\title{
Windschitl type approximation formulas for the gamma function
}

\section{Zhen-Hang Yang ${ }^{1,2}$ (D) and Jing-Feng Tian ${ }^{1 *}$ (D)}

\section{"Correspondence:}

tianjf@ncepu.edu.cn

${ }^{1}$ College of Science and

Technology, North China Electric

Power University, Baoding, P.R. China

Full list of author information is

available at the end of the article

\begin{abstract}
In this paper, we present four new Windschitl type approximation formulas for the gamma function. By some unique ideas and techniques, we prove that four functions combined with the gamma function and Windschitl type approximation formulas have good properties, such as monotonicity and convexity. These not only yield some new inequalities for the gamma and factorial functions, but also provide a new proof of known inequalities and strengthen known results.
\end{abstract}

MSC: Primary 41A10; 26A48; secondary 33B10; 26A51

Keywords: Gamma function; Windschitl type approximation formula; Monotonicity; Convexity; Inequality

\section{Introduction}

For $x>0$, the classical Euler's gamma function $\Gamma$ and psi (digamma) function $\psi$ are defined by

$$
\Gamma(x)=\int_{0}^{\infty} t^{x-1} e^{-t} d t \quad \text { and } \quad \psi(x)=\frac{\Gamma^{\prime}(x)}{\Gamma(x)},
$$

respectively. The derivatives $\psi^{\prime}, \psi^{\prime \prime}, \psi^{\prime \prime \prime}, \ldots$ are known as polygamma functions. The gamma function has various important applications in many branches of science. For this reason, scholars strive to find various better approximations for the factorial or gamma function by using different ideas and techniques, for instance, Ramanujan [1, p. 339], Burnside [2], Gosper [3], Alzer [4], Shi et al. [5], Batir [6, 7], Mortici [8-12], Nemes [13, Corollary 4.1], [14], Qi et al. [15, 16], Feng and Wang [17], Chen [18-21], Yang et al. [22-25], Lu et al. [26-28], Xu et al. [29]. Some properties of the remainders of certain approximations for the gamma function can be found in [4, 16, 23, 30-35].

In this paper, we are interested in Windschitl's approximation formula (see [36]) given by

$$
\Gamma(x+1) \sim W_{0}(x)=\sqrt{2 \pi x}\left(\frac{x}{e}\right)^{x}\left(x \sinh \frac{1}{x}\right)^{x / 2}, \quad \text { as } x \rightarrow \infty .
$$

(c) The Author(s) 2018. This article is distributed under the terms of the Creative Commons Attribution 4.0 International License (http://creativecommons.org/licenses/by/4.0/), which permits unrestricted use, distribution, and reproduction in any medium, provided you give appropriate credit to the original author(s) and the source, provide a link to the Creative Commons license, and indicate if changes were made. 
As shown in [21, Eq. (3.18)], the rate of Windschitl's approximation $W_{0}(x)$ converging to $\Gamma(x+1)$ is like $x^{-5}$ as $x \rightarrow \infty$, and like $x^{-7}$ if one replaces $W_{0}(x)$ with

$$
W_{1}(x)=\sqrt{2 \pi x}\left(\frac{x}{e}\right)^{x}\left(x \sinh \frac{1}{x}+\frac{1}{810 x^{6}}\right)^{x / 2}
$$

by an easy check. These show that $W_{0}(x)$ and $W_{1}(x)$ are more accurate approximations for the gamma function. In 2009, Alzer [37] proved that for all $x>0$,

$$
\begin{aligned}
\sqrt{2 \pi x}\left(\frac{x}{e}\right)^{x}\left(x \sinh \frac{1}{x}\right)^{x / 2}\left(1+\frac{\alpha}{x^{5}}\right) & <\Gamma(x+1) \\
& <\sqrt{2 \pi x}\left(\frac{x}{e}\right)^{x}\left(x \sinh \frac{1}{x}\right)^{x / 2}\left(1+\frac{\beta}{x^{5}}\right)
\end{aligned}
$$

with the best possible constants $\alpha=0$ and $\beta=1 / 1620$. Recently, Lu, Song and Ma [27] extended Windschitl's formula to an asymptotic expansion:

$$
\Gamma(n+1) \sim \sqrt{2 \pi n}\left(\frac{n}{e}\right)^{n}\left[n \sinh \left(\frac{1}{n}+\frac{a_{7}}{n^{7}}+\frac{a_{9}}{n^{9}}+\frac{a_{11}}{n^{11}}+\cdots\right)\right]^{n / 2}
$$

as $n \rightarrow \infty$ with $a_{7}=1 / 810, a_{9}=-67 / 42525, a_{11}=19 / 8505, \ldots$, and proved that there exists an $m$ such that, for every $x>m$, the double inequality

$$
\left[x \sinh \left(\frac{1}{x}+\frac{1}{810 x^{7}}-\frac{67}{42525 x^{9}}\right)\right]^{x / 2}<\frac{\Gamma(x+1)}{\sqrt{2 \pi x}(x / e)^{x}}<\left[x \sinh \left(\frac{1}{x}+\frac{1}{810 x^{7}}\right)\right]^{x / 2}
$$

holds. An explicit formula for determining the coefficients of $n^{-k}(n \in \mathbb{N})$ was given in [19, Theorem 1] by Chen. Another asymptotic expansion

$$
\Gamma(x+1) \sim \sqrt{2 \pi x}\left(\frac{x}{e}\right)^{x}\left(x \sinh \frac{1}{x}\right)^{x / 2+\sum_{j=0}^{\infty} r_{j} x^{-j}}, \quad \text { as } x \rightarrow \infty,
$$

was presented in the same paper [19, Theorem 2].

Let us consider the four new Windschitl type approximation formulas, as $x \rightarrow \infty$, which are

$$
\begin{aligned}
& \Gamma(x+1) \sim \sqrt{2 \pi x}\left(\frac{x}{e}\right)^{x}\left(x \sinh \frac{1}{x}\right)^{x / 2} \exp \left(\frac{1}{1620 x^{5}}\right):=W_{01}(x), \\
& \Gamma(x+1) \sim \sqrt{2 \pi x}\left(\frac{x}{e}\right)^{x}\left(x \sinh \frac{1}{x}\right)^{x / 2} \exp \left(\frac{1}{1620 x^{5}}-\frac{11}{18,900 x^{7}}\right):=W_{02}(x), \\
& \Gamma(x+1) \sim \sqrt{2 \pi x}\left(\frac{x}{e}\right)^{x}\left(x \sinh \frac{1}{x}\right)^{x / 2}\left(1+\frac{1}{1620 x^{5}}\right):=W_{01}^{*}(x), \\
& \Gamma(x+1) \sim \sqrt{2 \pi x}\left(\frac{x}{e}\right)^{x}\left(x \sinh \frac{1}{x}\right)^{x / 2}\left(1+\frac{1}{1620 x^{5}}-\frac{11}{18,900 x^{7}}\right)=W_{02}^{*}(x) .
\end{aligned}
$$

The aim of this paper is, by investigating the monotonicity and convexity of the functions

$$
x \mapsto \ln \Gamma(x+1)-\ln F(x), \quad \text { where } F=W_{01}, W_{02}, W_{01}^{*}, W_{02}^{*},
$$


to establish some new sharp inequalities between the gamma function $\Gamma(x+1)$ and Windschitl's approximation formula $W_{0}(x)$. As a by-product, a concise proof of Alzer inequalities (1.4) is presented, and a strengthening for Lu et al's inequalities (1.6) is given.

The rest of this paper is organized as follows. In Sect. 2, three lemmas are given, which are crucial to the proofs of our results. In Sect. 3, five monotonicity and convexity results for the functions constructed from the gamma function and Windschilt's formula are proved. Some new inequalities between the gamma or factorial functions with Windschilt's formula are established in Sect. 4. In Sect. 5, numeric comparisons of several better approximation formulas are presented.

\section{Lemmas}

To prove our results, we need three lemmas as follows.

Lemma 1 The inequalities

$$
\begin{aligned}
& x \frac{x^{2}+\frac{71}{84}}{x^{4}+\frac{13}{14} x^{2}+\frac{27}{560}}<\psi^{\prime}\left(x+\frac{1}{2}\right) \\
& x \frac{x^{4}+\frac{227}{66} x^{2}+\frac{4237}{2640}}{x^{6}+\frac{155}{44} x^{4}+\frac{329}{176} x^{2}+\frac{375}{4928}}<\psi^{\prime}\left(x+\frac{1}{2}\right)<\frac{1}{x} \frac{x^{4}+\frac{67}{36} x^{2}+\frac{256}{945}}{x^{4}+\frac{35}{18} x^{2}+\frac{407}{1008}}
\end{aligned}
$$

hold for $x>0$.

Proof The inequality (2.1) was proved in [38, Remark 2.2].

Let

$$
\begin{aligned}
& g_{1}(x)=\psi^{\prime}\left(x+\frac{1}{2}\right)-\frac{1}{x} \frac{x^{4}+\frac{67}{36} x^{2}+\frac{256}{945}}{x^{4}+\frac{35}{18} x^{2}+\frac{407}{1008}} \\
& g_{2}(x)=\psi^{\prime}\left(x+\frac{1}{2}\right)-x \frac{x^{4}+\frac{227}{66} x^{2}+\frac{4237}{2640}}{x^{6}+\frac{155}{44} x^{4}+\frac{329}{176} x^{2}+\frac{375}{4928}} .
\end{aligned}
$$

Then we have

$$
\begin{aligned}
g_{1}(x+1)-g_{1}(x)= & \psi^{\prime}\left(x+\frac{3}{2}\right)-\frac{1}{x+1} \frac{(x+1)^{4}+\frac{67}{36}(x+1)^{2}+\frac{256}{945}}{(x+1)^{4}+\frac{35}{18}(x+1)^{2}+\frac{407}{1008}} \\
& -\psi^{\prime}\left(x+\frac{1}{2}\right)+\frac{1}{x} \frac{x^{4}+\frac{67}{36} x^{2}+\frac{256}{945}}{x^{4}+\frac{35}{18} x^{2}+\frac{407}{1008}} \\
= & 921,600 \times\left[x(2 x+1)^{2}(x+1)\left(1008 x^{4}+1960 x^{2}+407\right)\right]^{-1} \\
& \times\left(1008 x^{4}+4032 x^{3}+8008 x^{2}+7952 x+3375\right)^{-1}>0 .
\end{aligned}
$$

Hence, we conclude that

$$
g_{1}(x)<g_{1}(x+1)<\cdots<\lim _{n \rightarrow \infty} g_{1}(x+n)=0
$$

which proves the first inequality of (2.2). 
Analogously, we have

$$
\begin{aligned}
& g_{2}(x+1)-g_{2}(x) \\
&=\psi^{\prime}\left(x+\frac{3}{2}\right)-\frac{(x+1)\left((x+1)^{4}+\frac{227}{66}(x+1)^{2}+\frac{4237}{2640}\right)}{(x+1)^{6}+\frac{155}{44}(x+1)^{4}+\frac{329}{176}(x+1)^{2}+\frac{375}{4928}} \\
& \quad-\psi^{\prime}\left(x+\frac{1}{2}\right)+\frac{x\left(x^{4}+\frac{227}{66} x^{2}+\frac{4237}{2640}\right)}{x^{6}+\frac{155}{44} x^{4}+\frac{329}{176} x^{2}+\frac{375}{4928}} \\
&=-58,982,400 \times\left[(2 x+1)^{2}\left(4928 x^{6}+17,360 x^{4}+9212 x^{2}+375\right)\right]^{-1} \\
& \quad \times\left(4928 x^{6}+29,568 x^{5}+91,280 x^{4}+168,000 x^{3}\right. \\
&\left.\quad+187,292 x^{2}+117,432 x+31,875\right)^{-1} \\
&< 0 .
\end{aligned}
$$

It then follows that

$$
g_{2}(x)>g_{2}(x+1)>\cdots>\lim _{n \rightarrow \infty} g_{2}(x+n)=0
$$

which proves the second formula of (2.2). This completes the proof.

Lemma 2 The inequalities

$$
\begin{aligned}
& \frac{t^{2}}{\sinh ^{2} t}>1-\frac{1}{3} t^{2}+\frac{1}{15} t^{4}-\frac{2}{189} t^{6} \\
& \frac{t^{2}}{\sinh ^{2} t}>1-\frac{1}{3} t^{2}+\frac{1}{15} t^{4}-\frac{2}{189} t^{6}+\frac{1}{675} t^{8}-\frac{2}{10,395} t^{10}
\end{aligned}
$$

hold for all $t>0$.

Proof The inequalities in question are equivalent to

$$
h_{1}(t)=\left(\frac{2}{189} t^{6}-\frac{1}{15} t^{4}+\frac{1}{3} t^{2}-1\right) \frac{\cosh 2 t-1}{2 t^{2}}+1>0
$$

and

$$
h_{2}(t)=\left(\frac{2}{10,395} t^{10}-\frac{1}{675} t^{8}+\frac{2}{189} t^{6}-\frac{1}{15} t^{4}+\frac{1}{3} t^{2}-1\right) \frac{\cosh 2 t-1}{2 t^{2}}+1>0
$$

for $t>0$, respectively.

Expanding into a power series yields

$$
\begin{aligned}
h_{1}(t) & =\left(\frac{2}{189} t^{6}-\frac{1}{15} t^{4}+\frac{1}{3} t^{2}-1\right) \sum_{n=0}^{\infty} \frac{2^{2 n+1}}{(2 n+2) !} t^{2 n}+1 \\
& =\frac{2}{189} \sum_{n=3}^{\infty} \frac{(2 t)^{2 n}}{32(2 n-4) !}-\frac{1}{15} \sum_{n=2}^{\infty} \frac{(2 t)^{2 n}}{8(2 n-2) !}+\frac{1}{3} \sum_{n=1}^{\infty} \frac{(2 t)^{2 n}}{2(2 n) !}
\end{aligned}
$$




$$
-2 \sum_{n=0}^{\infty} \frac{(2 t)^{2 n}}{(2 n+2) !}+1:=\frac{1}{1890} \sum_{n=3}^{\infty} \frac{(n-3) \times p_{5}(n)}{(2 n+2) !}(2 t)^{2 n}
$$

where

$$
p_{5}(n)=40 n^{5}+60 n^{4}-122 n^{3}-543 n^{2}-296 n+1050 .
$$

We assert that $p_{5}(n)>0$ for $n \geq 3$, since $p_{5}(n)$ can be written as

$$
p_{5}(n)=40(n-3)^{5}+660(n-3)^{4}+4198(n-3)^{3}+12,399(n-3)^{2}+15,832(n-3)+6561,
$$

which is evidently positive for $n \geq 3$. Hence $h_{1}(t)>0$ for all $t>0$. While

$$
\begin{aligned}
h_{2}(t)= & h_{1}(t)+\left(\frac{2}{10,395} t^{10}-\frac{1}{675} t^{8}\right) \sum_{n=0}^{\infty} \frac{2^{2 n+1}}{(2 n+2) !} t^{2 n} \\
= & \frac{1}{1890} \sum_{n=3}^{\infty} \frac{(n-3) \times p_{5}(n)}{(2 n+2) !}(2 t)^{2 n}+\frac{2}{10,395} \sum_{n=5}^{\infty} \frac{(2 t)^{2 n}}{2^{9}(2 n-8) !} \\
& -\frac{1}{675} \sum_{n=4}^{\infty} \frac{(2 t)^{2 n}}{2^{7}(2 n-6) !}:=\frac{1}{415,800} \sum_{n=5}^{\infty} \frac{(n-5) \times p_{9}(n)}{(2 n+2) !}(2 t)^{2 n},
\end{aligned}
$$

where

$$
\begin{aligned}
p_{9}(n)= & 160 n^{9}-1200 n^{8}+2368 n^{7}-1768 n^{6}+2354 n^{5}+14,845 n^{4} \\
& -6403 n^{3}-70,782 n^{2}-57,384 n+138,600 .
\end{aligned}
$$

It is easy to check that

$$
\begin{aligned}
p_{9}(n)= & 160 m^{9}+6000 m^{8}+98,368 m^{7}+921,112 m^{6}+5392,514 m^{5}+20,270,695 m^{4} \\
& +48,258,997 m^{3}+68,827,423 m^{2}+51,883,321 m+15,041,130>0,
\end{aligned}
$$

for $m=n-5 \geq 0$, which proves $h_{2}(t)>0$ for $t>0$. The proof is complete.

The following lemma offers a simple criterion to determine the sign of a class of special polynomials on given interval contained in $(0, \infty)$ without using Descartes' Rule of Signs, which plays an important role in studying certain special functions, see, for example, [39, 40]. A series version can be found in [41, 42].

Lemma 3 ([39, Lemma 7]) Let $n \in \mathbb{N}$ and $m \in \mathbb{N} \cup\{0\}$ with $n>m$ and let $P_{n}(t)$ be an $n$th degree polynomial defined by

$$
P_{n}(t)=\sum_{i=m+1}^{n} a_{i} t^{i}-\sum_{i=0}^{m} a_{i} t^{i}
$$

where $a_{n}, a_{m}>0, a_{i} \geq 0$ for $0 \leq i \leq n-1$ with $i \neq m$. Then there is a unique number $t_{m+1} \in(0, \infty)$ satisfying $P_{n}\left(t_{m+1}\right)=0$ such that $P_{n}(t)<0$ for $t \in\left(0, t_{m+1}\right)$ and $P_{n}(t)>0$ for $t \in\left(t_{m+1}, \infty\right)$. 
Consequently, for a given $t_{0}>0$, if $P_{n}\left(t_{0}\right)>0$ then $P_{n}(t)>0$ for $t \in\left(t_{0}, \infty\right)$ and if $P_{n}\left(t_{0}\right)<0$ then $P_{n}(t)<0$ for $t \in\left(0, t_{0}\right)$.

\section{Monotonicity and convexity}

Theorem 1 The function

$$
f_{0}(x)=\ln \Gamma(x+1)-\ln \sqrt{2 \pi}-\left(x+\frac{1}{2}\right) \ln x+x-\frac{x}{2} \ln \left(x \sinh \frac{1}{x}\right)
$$

is strictly decreasing and convex on $(0, \infty)$.

Proof Differentiation yields

$$
\begin{aligned}
& f_{0}^{\prime}(x)=\psi(x+1)-\frac{1}{2} \ln \left(x \sinh \frac{1}{x}\right)+\frac{1}{2 x} \operatorname{coth} \frac{1}{x}-\ln x-\frac{1}{2 x}-\frac{1}{2} \\
& f_{0}^{\prime \prime}(x)=\psi^{\prime}(x+1)+\frac{1}{2 x^{3}} \frac{1}{\sinh ^{2}(1 / x)}-\frac{3}{2 x}+\frac{1}{2 x^{2}} .
\end{aligned}
$$

Replacing $x$ by $(x+1 / 2)$ in inequality (2.1) leads to

$$
\psi^{\prime}(x+1)>\frac{5}{6} \frac{(2 x+1)\left(21 x^{2}+21 x+23\right)}{35 x^{4}+70 x^{3}+85 x^{2}+50 x+12}
$$

and using which to $f_{0}^{\prime \prime}(x)$ gives

$$
\begin{aligned}
f_{0}^{\prime \prime}(x)> & \frac{5}{6} \frac{(2 x+1)\left(21 x^{2}+21 x+23\right)}{35 x^{4}+70 x^{3}+85 x^{2}+50 x+12} \\
& +\frac{1}{2 x^{3}} \frac{1}{\sinh ^{2}(1 / x)}-\frac{3}{2 x}+\frac{1}{2 x^{2}}=f_{01}\left(\frac{1}{x}\right) .
\end{aligned}
$$

Simplifying yields

$$
\begin{aligned}
f_{01}(t) & =\frac{1}{2} \frac{t^{3}}{\sinh ^{2} t}+\frac{1}{6} \frac{t\left(36 t^{5}+42 t^{4}-80 t^{3}-220 t^{2}-210 t-105\right)}{12 t^{4}+50 t^{3}+85 t^{2}+70 t+35} \\
& =\frac{t}{12} \frac{f_{02}(t)}{\left(12 t^{4}+50 t^{3}+85 t^{2}+70 t+35\right) \sinh ^{2} t},
\end{aligned}
$$

where

$$
\begin{aligned}
f_{02}(t)= & \left(36 t^{5}+42 t^{4}-80 t^{3}-220 t^{2}-210 t-105\right) \cosh 2 t \\
& +\left(72 t^{6}+264 t^{5}+468 t^{4}+500 t^{3}+430 t^{2}+210 t+105\right) .
\end{aligned}
$$

Expanding into a power series gives

$$
\begin{aligned}
f_{02}(t)= & \left(36 \sum_{n=2}^{\infty} \frac{2^{2 n-4}}{(2 n-4) !} t^{2 n+1}-80 \sum_{n=1}^{\infty} \frac{2^{2 n-2}}{(2 n-2) !} t^{2 n+1}-210 \sum_{n=0}^{\infty} \frac{2^{2 n}}{(2 n) !} t^{2 n+1}\right) \\
& +\left(42 \sum_{n=2}^{\infty} \frac{2^{2 n-4}}{(2 n-4) !} t^{2 n}-220 \sum_{n=1}^{\infty} \frac{2^{2 n-2}}{(2 n-2) !} t^{2 n}-105 \sum_{n=0}^{\infty} \frac{2^{2 n}}{(2 n) !} t^{2 n}\right) \\
& +\left(72 t^{6}+264 t^{5}+468 t^{4}+500 t^{3}+430 t^{2}+210 t+105\right)
\end{aligned}
$$




$$
\begin{aligned}
= & \sum_{n=4}^{\infty} \frac{(n-3)\left(36 n^{3}+19 n+70\right) 2^{2 n}}{(2 n) !} t^{2 n+1} \\
& +\sum_{n=4}^{\infty} \frac{\left(84 n^{4}-252 n^{3}-209 n^{2}+157 n-210\right) 2^{2 n-2}}{(2 n) !} t^{2 n}>0,
\end{aligned}
$$

where the inequality holds due to

$$
\begin{aligned}
& 84 n^{4}-252 n^{3}-209 n^{2}+157 n-210 \\
& \quad=84(n-4)^{4}+1092(n-4)^{3}+4831(n-4)^{2}+7893(n-4)+2450>0
\end{aligned}
$$

for $n \geq 4$.

It then follows that $f_{01}(t)>0$ for $t>0$, so $f_{0}^{\prime \prime}(x)>0$ for $x>0$. This yields $f_{0}^{\prime}(x)<$ $\lim _{x \rightarrow \infty} f_{0}^{\prime}(x)=0$, which proves the desired result.

Theorem 2 The function

$$
f_{1}^{*}(x)=\ln \Gamma(x+1)-\ln \sqrt{2 \pi}-\left(x+\frac{1}{2}\right) \ln x+x-\frac{x}{2} \ln \left(x \sinh \frac{1}{x}\right)-\ln \left(1+\frac{1}{1620 x^{5}}\right)
$$

is strictly increasing and concave on $(0, \infty)$.

Proof Differentiation yields

$$
\begin{aligned}
f_{1}^{* \prime}(x)= & \psi(x+1)-\frac{1}{2} \ln \left(x \sinh \frac{1}{x}\right)+\frac{1}{2 x} \operatorname{coth} \frac{1}{x} \\
& -\ln x-\frac{1}{2 x}-\frac{1}{2}+\frac{5}{x\left(1620 x^{5}+1\right)} \\
f_{1}^{* \prime \prime}(x)= & \psi^{\prime}(x+1)+\frac{1}{2 x^{3}} \frac{1}{\sinh ^{2}(1 / x)}-\frac{3}{2 x}+\frac{1}{2 x^{2}}-5 \frac{9720 x^{5}+1}{x^{2}\left(1620 x^{5}+1\right)^{2}} .
\end{aligned}
$$

Since $\lim _{x \rightarrow \infty} f_{1}^{* \prime}(x)=0$, it suffices to prove $f_{1}^{* \prime \prime}(x)<0$ for $x>0$. Replacing $x$ by $(x+1 / 2)$ in the right-hand side inequality of (2.2) leads to

$$
\psi^{\prime}(x+1)<\frac{1}{30} \frac{3780 x^{4}+7560 x^{3}+12,705 x^{2}+8925 x+3019}{(2 x+1)\left(63 x^{4}+126 x^{3}+217 x^{2}+154 x+60\right)},
$$

which indicates that

$$
\begin{aligned}
f_{1}^{* \prime \prime}(x)< & \frac{1}{30} \frac{3780 x^{4}+7560 x^{3}+12,705 x^{2}+8925 x+3019}{(2 x+1)\left(63 x^{4}+126 x^{3}+217 x^{2}+154 x+60\right)} \\
& +\frac{1}{2 x^{3}} \frac{1}{\sinh ^{2}(1 / x)}-\frac{3}{2 x}+\frac{1}{2 x^{2}}-5 \frac{9720 x^{5}+1}{x^{2}\left(1620 x^{5}+1\right)^{2}}:=f_{11}^{*}\left(\frac{1}{x}\right)
\end{aligned}
$$

where

$$
\begin{aligned}
f_{11}^{*}(t)= & \frac{t^{3}}{\cosh 2 t-1}-\frac{3}{2} t+\frac{1}{2} t^{2}-5 t^{7} \frac{t^{5}+9720}{\left(t^{5}+1620\right)^{2}} \\
& +\frac{1}{30} \frac{t\left(3019 t^{4}+8925 t^{3}+12,705 t^{2}+7560 t+3780\right)}{(t+2)\left(60 t^{4}+154 t^{3}+217 t^{2}+126 t+63\right)} .
\end{aligned}
$$


Using the inequality

$$
\cosh 2 t-1>\sum_{n=1}^{4} \frac{2^{2 n}}{(2 n) !} t^{2 n}=2 t^{2}+\frac{2}{3} t^{4}+\frac{4}{45} t^{6}+\frac{2}{315} t^{8}
$$

yields

$$
\begin{aligned}
f_{11}^{*}( & t) \\
< & \frac{t^{3}}{2 t^{2}+\frac{2}{3} t^{4}+\frac{4}{45} t^{6}+\frac{2}{315} t^{8}}-\frac{3}{2} t+\frac{1}{2} t^{2}-5 t^{7} \frac{t^{5}+9720}{\left(t^{5}+1620\right)^{2}} \\
& +\frac{1}{30} \frac{t\left(3019 t^{4}+8925 t^{3}+12,705 t^{2}+7560 t+3780\right)}{(t+2)\left(60 t^{4}+154 t^{3}+217 t^{2}+126 t+63\right)} \\
= & -\frac{1}{30} \\
& \times \frac{t^{9} \times f_{12}^{*}(t)}{\left(t^{5}+1620\right)^{2}(t+2)\left(t^{6}+14 t^{4}+105 t^{2}+315\right)\left(60 t^{4}+154 t^{3}+217 t^{2}+126 t+63\right)} \\
< & 0
\end{aligned}
$$

for $t>0$, where the inequality holds due to

$$
\begin{aligned}
f_{12}^{*}(t)= & 8100 t^{14}+39,690 t^{13}+193,586 t^{12}+645,960 t^{11}+2,028,124 t^{10} \\
& +90,019,275 t^{9}+406,666,800 t^{8}+1976,029,740 t^{7}+6395,589,900 t^{6} \\
& +20,173,546,260 t^{5}+51,035,406,750 t^{4}+110,592,337,500 t^{3} \\
& +184,843,490,400 t^{2}+254,068,164,000 t+101,627,265,600>0
\end{aligned}
$$

for $t>0$. This implies that $f_{2}^{\prime \prime}(x)<0$ for all $x>0$, and the proof is complete.

\section{Theorem 3 The function}

$$
f_{1}(x)=\ln \Gamma(x+1)-\ln \sqrt{2 \pi}-\left(x+\frac{1}{2}\right) \ln x+x-\frac{x}{2} \ln \left(x \sinh \frac{1}{x}\right)-\frac{1}{1620 x^{5}}
$$

is strictly increasing and concave on $(0, \infty)$.

Proof We clearly see that

$$
f_{1}(x)=f_{1}^{*}(x)+D\left(\frac{1}{1620 x^{5}}\right)
$$

where $D(y)=\ln (1+y)-y$. By Theorem $2, f_{1}^{*}$ is strictly increasing and concave on $(0, \infty)$, so if we prove $x \mapsto D(y)$ is strictly increasing and concave on $(0, \infty)$, then so will be $f_{1}$, and the proof will be complete. Now we easily check that for $x>0$,

$$
\frac{d D(y)}{d x}=\frac{1}{324 x^{6}\left(1620 x^{5}+1\right)}>0,
$$




$$
\frac{d^{2} D(y)}{d x^{2}}=-\frac{1}{54} \frac{2970 x^{5}+1}{x^{7}\left(1620 x^{5}+1\right)^{2}}<0
$$

which completes the proof.

Theorem 4 The function

$$
\begin{aligned}
f_{2}(x)= & \ln \Gamma(x+1)-\ln \sqrt{2 \pi}-\left(x+\frac{1}{2}\right) \ln x+x \\
& -\frac{x}{2} \ln \left(x \sinh \frac{1}{x}\right)-\frac{1}{1620 x^{5}}+\frac{11}{18,900 x^{7}}
\end{aligned}
$$

is strictly decreasing and convex on $(0, \infty)$.

Proof Differentiation yields

$$
\begin{aligned}
f_{2}^{\prime}(x)= & \psi(x+1)-\frac{1}{2} \ln \left(x \sinh \frac{1}{x}\right)+\frac{1}{2 x} \operatorname{coth} \frac{1}{x} \\
& -\ln x-\frac{1}{2 x}-\frac{1}{2}+\frac{1}{324 x^{6}}-\frac{11}{2700 x^{8}} \\
f_{2}^{\prime \prime}(x)= & \psi^{\prime}(x+1)+\frac{1}{2 x^{3}} \frac{1}{\sinh ^{2}(1 / x)}-\frac{3}{2 x}+\frac{1}{2 x^{2}}-\frac{1}{54 x^{7}}+\frac{22}{675 x^{9}} .
\end{aligned}
$$

Since $\lim _{x \rightarrow \infty} f_{2}^{\prime}(x)=0$, it suffices to prove $f_{2}^{\prime \prime}(x)>0$ for $x>0$. Replacing $x$ by $(x+1 / 2)$ in the left-hand side inequality of (2.2) leads to

$$
\psi^{\prime}(x+1)>\frac{7}{30} \frac{(2 x+1)\left(165 x^{4}+330 x^{3}+815 x^{2}+650 x+417\right)}{77 x^{6}+231 x^{5}+560 x^{4}+735 x^{3}+623 x^{2}+294 x+60},
$$

and applying which to $f_{2}^{\prime \prime}(x)$ gives

$$
\begin{aligned}
f_{2}^{\prime \prime}(x)> & \frac{7}{30} \frac{(2 x+1)\left(165 x^{4}+330 x^{3}+815 x^{2}+650 x+417\right)}{77 x^{6}+231 x^{5}+560 x^{4}+735 x^{3}+623 x^{2}+294 x+60} \\
& +\frac{1}{2 x^{3}} \frac{1}{\sinh ^{2}(1 / x)}-\frac{3}{2 x}+\frac{1}{2 x^{2}}-\frac{1}{54 x^{7}}+\frac{22}{675 x^{9}}=f_{21}\left(\frac{1}{x}\right)
\end{aligned}
$$

Making a change of variable $t=1 / x$ yields

$$
\begin{aligned}
f_{21}(t)= & \frac{7}{30} \frac{t(t+2)\left(417 t^{4}+650 t^{3}+815 t^{2}+330 t+165\right)}{60 t^{6}+294 t^{5}+623 t^{4}+735 t^{3}+560 t^{2}+231 t+77} \\
& -\frac{3}{2} t+\frac{1}{2} t^{2}-\frac{1}{54} t^{7}+\frac{22}{675} t^{9}+\frac{t}{2} \frac{t^{2}}{\sinh ^{2} t} .
\end{aligned}
$$

We distinguish two cases to prove $f_{21}(t)>0$ for all $t>0$.

Case 1: $t \geq 1$. Application of inequality (2.3) gives

$$
\begin{aligned}
f_{21}(t)> & \frac{7}{30} \frac{t(t+2)\left(417 t^{4}+650 t^{3}+815 t^{2}+330 t+165\right)}{60 t^{6}+294 t^{5}+623 t^{4}+735 t^{3}+560 t^{2}+231 t+77}-\frac{3}{2} t+\frac{1}{2} t^{2} \\
& -\frac{1}{54} t^{7}+\frac{22}{675} t^{9}+\frac{t}{2}\left(1-\frac{1}{3} t^{2}+\frac{1}{15} t^{4}-\frac{2}{189} t^{6}\right)
\end{aligned}
$$




$$
=\frac{1}{9450} \frac{t^{9} \times p_{6}(t)}{60 t^{6}+294 t^{5}+623 t^{4}+735 t^{3}+560 t^{2}+231 t+77},
$$

where

$$
p_{6}(t)=18,480 t^{6}+90,552 t^{5}+178,384 t^{4}+160,230 t^{3}+51,205 t^{2}-1617 t-539 .
$$

Clearly, $p_{6}(t)>0$ for $t \geq 1$, so $f_{21}(t)>0$ for $t \geq 1$.

Case 2: $0<t<1$. Using inequality (2.4) yields

$$
\begin{aligned}
f_{21}(t)> & \frac{7}{30} \frac{t(t+2)\left(417 t^{4}+650 t^{3}+815 t^{2}+330 t+165\right)}{60 t^{6}+294 t^{5}+623 t^{4}+735 t^{3}+560 t^{2}+231 t+77}-\frac{3}{2} t+\frac{1}{2} t^{2} \\
& -\frac{1}{54} t^{7}+\frac{22}{675} t^{9}+\frac{t}{2}\left(1-\frac{1}{3} t^{2}+\frac{1}{15} t^{4}-\frac{2}{189} t^{6}+\frac{1}{675} t^{8}-\frac{2}{10,395} t^{10}\right) \\
= & \frac{t^{11} \times q_{6}(t)}{60 t^{6}+294 t^{5}+623 t^{4}+735 t^{3}+560 t^{2}+231 t+77},
\end{aligned}
$$

where

$$
q_{6}(t)=-\frac{4}{693} t^{6}-\frac{14}{495} t^{5}+\frac{2881}{1485} t^{4}+\frac{4816}{495} t^{3}+\frac{400,919}{20,790} t^{2}+\frac{1573}{90} t+\frac{1573}{270} .
$$

Since the coefficients of polynomial $q_{6}(t)$ satisfy the conditions of Lemma 3 and $q_{6}(1)=$ $53,681 / 990>0$, we find that $q_{6}(t)>0$ for $t \in(0,1)$, and then $f_{21}(t)>0$ for $t \in(0,1)$.

This ends the proof.

\section{Theorem 5 The function}

$$
\begin{aligned}
f_{2}^{*}(x)= & \ln \Gamma(x+1)-\ln \sqrt{2 \pi}-\left(x+\frac{1}{2}\right) \ln x+x \\
& -\frac{x}{2} \ln \left(x \sinh \frac{1}{x}\right)-\ln \left(1+\frac{1}{1620 x^{5}}-\frac{11}{18,900 x^{7}}\right)
\end{aligned}
$$

is strictly decreasing and convex on $[4 / 3, \infty)$.

Proof We easily see that

$$
f_{2}^{*}(x)=f_{2}(x)-D\left(\frac{1}{1620 x^{5}}-\frac{11}{18,900 x^{7}}\right)
$$

where $D(y)=\ln (1+y)-y$. By Theorem $4, f_{2}$ is strictly decreasing and convex on $(0, \infty)$, so if we prove $x \mapsto D(y)$ is strictly increasing and concave on $[4 / 3, \infty)$, then so will be $f_{2}^{*}$, and the proof will be complete. Now we easily check that for $x \geq 4 / 3$,

$$
\begin{aligned}
& \frac{d D(y)}{d x}=\frac{1}{8100} \frac{\left(25 x^{2}-33\right)\left(35 x^{2}-33\right)}{x^{8}\left(35 x^{2}+56,700 x^{7}-33\right)}>0, \\
& \frac{d^{2} D(y)}{d x^{2}}=-\frac{p_{11}(x)}{x^{9}\left(56,700 x^{7}+35 x^{2}-33\right)^{2}}<0,
\end{aligned}
$$


where the last inequality holds due to

$$
\begin{aligned}
p_{11}(x)= & 67,375 x^{11}-180,180 x^{9}+114,345 x^{7}+\frac{1225}{54} x^{6}-\frac{2233}{27} x^{4}+\frac{8591}{90} x^{2}-\frac{2662}{75} \\
= & 385 x^{7}\left[\left(175\left(x^{2}-\frac{16}{9}\right)^{2}+\frac{1388}{9}\left(x^{2}-\frac{16}{9}\right)+\frac{1465}{81}\right)\right] \\
& +\frac{1}{1350} x^{2}\left(175 x^{2}-319\right)^{2}+\frac{242}{675}\left[56\left(x^{2}-\frac{16}{9}\right)+\frac{5}{9}\right]>0,
\end{aligned}
$$

which completes the proof.

\section{Inequalities}

As is well known, analytic inequalities [43-45] play a very important role in different branches of modern mathematics. Using the theorems presented in the previous section, we can obtain some new inequalities for the gamma function and factorial function related to Windschitl's formula.

Corollary 1 Let $W_{0}(x)$ be defined by (1.2). Then the inequalities

$$
\max \left(1, \exp \left(\frac{1}{1620 x^{5}}-\frac{11}{18,900 x^{7}}\right)\right)<\frac{\Gamma(x+1)}{W_{0}(x)}<1+\frac{1}{1620 x^{5}}<\exp \left(\frac{1}{1620 x^{5}}\right)
$$

hold for all $x>0$. If $x \geq \sqrt{33 / 35}$, then we have

$$
\begin{aligned}
1+\frac{1}{1620 x^{5}}-\frac{11}{18,900 x^{7}} & <\exp \left(\frac{1}{1620 x^{5}}-\frac{11}{18,900 x^{7}}\right) \\
& <\frac{\Gamma(x+1)}{W_{0}(x)}<1+\frac{1}{1620 x^{5}}<\exp \left(\frac{1}{1620 x^{5}}\right) .
\end{aligned}
$$

Proof The first and second inequalities in (4.1) follow directly from the monotonicity of $f_{0}, f_{2}$ and $f_{1}^{*}$ on $(0, \infty)$ given in Theorems 1,4 and 2 , respectively, due to $f_{0}(\infty)=f_{2}(\infty)=$ $f_{1}^{*}(\infty)=0$. The third one holds due to a simple inequality $1+y<e^{y}$ for $y>0$. The proof of inequalities (4.2) is similar, which completes the proof.

Using the monotonicity of $f_{0}, f_{1}^{*}$ and $f_{2}$ on $(0, \infty)$ and noting that

$$
f_{0}(1)=\ln \frac{e}{\sqrt{2 \pi \sinh 1}}, \quad f_{1}^{*}(1)=\ln \left(\frac{1620}{1621} \frac{e}{\sqrt{2 \pi \sinh 1}}\right), \quad f_{2}(1)=\ln \frac{e^{28,349 / 28,350}}{\sqrt{2 \pi \sinh 1}}
$$

we immediately get the following corollary.

Corollary 2 For $n \in \mathbb{N}$, the inequalities

$$
\begin{aligned}
& 1<\frac{n !}{\sqrt{2 \pi n}\left(\frac{n}{e}\right)^{n}\left(n \sinh \frac{1}{n}\right)^{n / 2}} \leq \alpha_{0}, \\
& \beta_{1}^{*}\left(1+\frac{1}{1620 n^{5}}\right) \leq \frac{n !}{\sqrt{2 \pi n}\left(\frac{n}{e}\right)^{n}\left(n \sinh \frac{1}{n}\right)^{n / 2}}<1+\frac{1}{1620 n^{5}},
\end{aligned}
$$




$$
\exp \left(\frac{1}{1620 n^{5}}-\frac{11}{18,900 n^{7}}\right)<\frac{n !}{\sqrt{2 \pi n}\left(\frac{n}{e}\right)^{n}\left(n \sinh \frac{1}{n}\right)^{n / 2}} \leq \alpha_{2} \exp \left(\frac{1}{1620 n^{5}}-\frac{11}{18,900 n^{7}}\right)
$$

hold with the best constants $\alpha_{0}=e / \sqrt{2 \pi \sinh 1} \approx 1.000,34, \beta_{1}^{*}=1620 e /(1621 \sqrt{2 \pi \sinh 1}) \approx$ $0.999,72$ and $\alpha_{2}=e^{28,349 / 28,350} / \sqrt{2 \pi \sinh 1} \approx 1.000,30$.

The proof of inequalities (1.4) presented by Alzer [37] seems to be somewhat complicated. With the aid of the first and second inequalities in (4.1), we can give a new and simpler proof.

Proof of inequalities (1.4) The sufficiency for the inequalities (1.4) to hold for $x>0$ follows by the first and second inequalities in (4.1). The necessary condition for the left-hand side inequality of (1.4) to hold for $x>0$ follows from the following relation:

$$
\begin{aligned}
& \lim _{x \rightarrow 0^{+}} \frac{\ln \Gamma(x+1)-\ln \sqrt{2 \pi}-\left(x+\frac{1}{2}\right) \ln x+x-\frac{x}{2} \ln \left(x \sinh \frac{1}{x}\right)-\ln \left(1+\frac{\alpha}{x^{5}}\right)}{\ln (1 / x)} \\
& \quad= \begin{cases}\frac{1}{2} & \text { if } \alpha=0, \\
-\frac{9}{2} & \text { if } \alpha \neq 0 .\end{cases}
\end{aligned}
$$

While the necessary condition for the right-hand side of (1.4) to hold for $x>0$ follows from the limit relation

$$
\begin{aligned}
& \lim _{x \rightarrow \infty} \frac{\ln \Gamma(x+1)-\ln \sqrt{2 \pi}-\left(x+\frac{1}{2}\right) \ln x+x-\frac{x}{2} \ln \left(x \sinh \frac{1}{x}\right)-\ln \left(1+\frac{\beta}{x^{5}}\right)}{x^{-5}} \\
& \quad=\frac{1}{1620}-\beta \leq 0 .
\end{aligned}
$$

This completes the proof.

The following corollary offers a strengthening for Lu et al's inequalities (1.6).

\section{Corollary 3 The inequalities}

$$
\begin{aligned}
{\left[x \sinh \left(\frac{1}{x}+\frac{1}{810 x^{7}}-\frac{67}{42,525 x^{9}}\right)\right]^{x / 2} } & <\left(x \sinh \frac{1}{x}\right)^{x / 2} \exp \left(\frac{1}{1620 x^{5}}-\frac{11}{18,900 x^{7}}\right) \\
& <\frac{\Gamma(x+1)}{\sqrt{2 \pi x}(x / e)^{x}} \\
& <\left(x \sinh \frac{1}{x}\right)^{x / 2} \exp \left(\frac{1}{1620 x^{5}}\right) \\
& <\left[x \sinh \left(\frac{1}{x}+\frac{1}{810 x^{7}}\right)\right]^{x / 2}
\end{aligned}
$$

hold for $x>c$, where $c=0$ for the second, third and fourth inequalities, while $c=x_{0} \approx$ 0.43738 for the first one, here $x_{0}$ is the unique solution of the equation

$$
\frac{1}{x}+\frac{1}{810 x^{7}}-\frac{67}{42,525 x^{9}}=0
$$

on $(0, \infty)$. 
Proof Clearly, the second and third inequalities of (4.3) follow by the first and second inequalities in (4.1). It remains to prove the first and last inequalities of (4.3).

(i) The last one is equivalent to

$$
\frac{x}{2} \ln \left[x \sinh \left(\frac{1}{x}+\frac{1}{810 x^{7}}\right)\right]>\frac{x}{2} \ln \left(x \sinh \frac{1}{x}\right)+\frac{1}{1620 x^{5}},
$$

or equivalently,

$$
h_{3}(t)=\ln \sinh \left(t+\frac{1}{810} t^{7}\right)-\ln \sinh t-\frac{1}{810} t^{6}>0
$$

for $t=1 / x>0$. Denote by $l(t)=\ln \sinh t$ and $t_{2}=\left(t+t^{7} / 810\right)$. Then by Taylor formula we have

$$
h_{3}(t)=l\left(t_{2}\right)-l(t)-\frac{1}{810} t^{6}=\left(t_{2}-t\right) l^{\prime}(t)+\frac{l^{\prime \prime}(t)}{2 !}\left(t_{2}-t\right)^{2}+\frac{l^{\prime \prime \prime}(\xi)}{3 !}\left(t_{2}-t\right)^{3}-\frac{1}{810} t^{6},
$$

where $t<\xi<t+t^{7} / 810$. Since $l^{\prime \prime \prime}(t)=2(\cosh t) / \sinh ^{3} t>0$, we get

$$
h_{3}(t)>\frac{1}{810} t^{7} \frac{\cosh t}{\sinh t}-\frac{t^{14}}{2 \times 810^{2}} \frac{1}{\sinh ^{2} t}-\frac{1}{810} t^{6}:=\frac{t^{6} \times h_{31}(t)}{2 \times 810^{2} \sinh ^{2} t},
$$

where

$$
h_{31}(t)=810 t \sinh 2 t-810 \cosh 2 t+810-t^{8} .
$$

Due to

$$
h_{31}(t)=540 t^{4}+144 t^{6}+\frac{101}{7} t^{8}+810 \sum_{n=5}^{\infty} \frac{(n-1) 2^{2 n}}{(2 n) !} t^{2 n}>0,
$$

we conclude that $h_{3}(t)>0$ for $t>0$.

(ii) To ensure that the first inequality holds, it is necessary to establish

$$
x \sinh \left(\frac{1}{x}+\frac{1}{810 x^{7}}-\frac{67}{42,525 x^{9}}\right)>0
$$

for $x>0$, for which it suffices so show that

$$
\frac{1}{x}+\frac{1}{810 x^{7}}-\frac{67}{42,525 x^{9}}=\frac{85,050 x^{8}+105 x^{2}-134}{85,050 x^{9}}>0 .
$$

By Lemma 3, the numerator in the above fraction, as an 8th degree polynomial, has a unique zero $x_{0}$ on $(0, \infty)$. Numeric computation gives $x_{0} \approx 0.437,38$.

Now the first inequality is equivalent to

$$
\frac{x}{2} \ln \left[x \sinh \left(\frac{1}{x}+\frac{1}{810 x^{7}}-\frac{67}{42,525 x^{9}}\right)\right]<\frac{x}{2} \ln \left(x \sinh \frac{1}{x}\right)+\frac{1}{1620 x^{5}}-\frac{11}{18,900 x^{7}}
$$


or equivalently,

$$
h_{4}(t)=\ln \left[\sinh \left(t+\frac{1}{810} t^{7}-\frac{67}{42,525} t^{9}\right)\right]-\ln (\sinh t)-\frac{1}{810} t^{6}+\frac{11}{9450} t^{8}<0
$$

for $t=1 / x \in\left(0,1 / x_{0}\right)$, where $1 / x_{0} \approx 2.28632$ is clearly the unique zero of the polynomial

$$
t_{1} \equiv t_{1}(t)=t+\frac{1}{810} t^{7}-\frac{67}{42,525} t^{9}
$$

on $(0, \infty)$. In view of $l^{\prime \prime}(t)=-1 / \sinh ^{2} t<0$, we have

$$
\begin{aligned}
h_{4}(t) & =l\left(t_{1}\right)-l(t)-\frac{1}{810} t^{6}+\frac{11}{9450} t^{8}<\left(t_{1}-t\right) l^{\prime}(t)-\frac{1}{810} t^{6}+\frac{11}{9450} t^{8} \\
& =\left(\frac{1}{810} t^{7}-\frac{67}{42,525} t^{9}\right) \frac{\cosh t}{\sinh t}-\frac{1}{810} t^{6}+\frac{11}{9450} t^{8} \\
& =-\frac{1}{85,050} \frac{t^{6}}{\sinh t}\left(105 \sinh t-105 t \cosh t+134 t^{3} \cosh t-99 t^{2} \sinh t\right) \\
& =-\frac{1}{85,050} \frac{t^{6}}{\sinh t} \sum_{n=3}^{\infty} \frac{4(268 n-99)(n-1)(n-2)}{(2 n-1) !} t^{2 n-1}<0,
\end{aligned}
$$

which completes the proof.

Remark 1 Clearly, the proof of Corollary 3 can also be regarded as a new proof of Lu et al.'s inequalities (1.6). Moreover, our proof gives the minimum value of $m$, i.e., $\min (m)=$ $x_{0} \approx 0.437,38$, such that the the double inequality (1.6) holds for all $x>x_{0}$.

\section{Numeric comparisons}

By the asymptotic expansion listed in [46, Eq. (6.1.40)]

$$
\ln \Gamma(x+1) \sim \ln \sqrt{2 \pi}+\left(x+\frac{1}{2}\right) \ln x-x+\sum_{n=1}^{\infty} \frac{B_{2 n}}{2 n(2 n-1) x^{2 n-1}}
$$

we easily verify that our four approximation formulas $W_{01}(x), W_{01}^{*}(x), W_{02}(x)$ and $W_{02}^{*}(x)$, defined by (1.8), (1.10), (1.9) and (1.11), respectively, have the following limit relations:

$$
\begin{aligned}
& \lim _{x \rightarrow \infty} \frac{\ln \Gamma(x+1)-\ln W_{01}(x)}{x^{-7}}=\lim _{x \rightarrow \infty} \frac{\ln \Gamma(x+1)-\ln W_{01}^{*}(x)}{x^{-7}}=-\frac{198}{340,200}, \\
& \lim _{x \rightarrow \infty} \frac{\ln \Gamma(x+1)-\ln W_{02}(x)}{x^{-9}}=\lim _{x \rightarrow \infty} \frac{\ln \Gamma(x+1)-\ln W_{02}^{*}(x)}{x^{-9}}=\frac{143}{170,100} .
\end{aligned}
$$

Also, for another approximation formula $W_{1}(x)$ defined by (1.3), we have

$$
\lim _{x \rightarrow \infty} \frac{\ln \Gamma(x+1)-\ln W_{1}(x)}{x^{-7}}=-\frac{163}{340,200} .
$$

Denote the two approximation formulas generated by the double inequality (1.6) by

$$
W_{L 1}(x)=\sqrt{2 \pi x}\left(\frac{x}{e}\right)^{x}\left[x \sinh \left(\frac{1}{x}+\frac{1}{810 x^{7}}\right)\right]^{x / 2} \text {, }
$$


Table 1 Comparison among $W_{02}(1.9), W_{L 2}(5.3), W_{1}(1.3), W_{01}^{*}(1.10)$ and $W_{L 1}$ (5.2)

\begin{tabular}{rlllll}
\hline$n$ & $\left|\frac{W_{02}(n)-n !}{n !}\right|$ & $\left|\frac{W_{L 2}(n)-n !}{n !}\right|$ & $\left|\frac{W_{1}(x)-n !}{n !}\right|$ & $\left|\frac{W_{01}^{*}(x)-n !}{n !}\right|$ & $\left|\frac{W_{L 1}(x)-n !}{n !}\right|$ \\
\hline 1 & $3.065 \times 10^{-4}$ & $5.655 \times 10^{-4}$ & $1.832 \times 10^{-4}$ & $2.754 \times 10^{-4}$ & $4.686 \times 10^{-4}$ \\
2 & $1.098 \times 10^{-6}$ & $1.629 \times 10^{-6}$ & $2.668 \times 10^{-6}$ & $3.449 \times 10^{-6}$ & $5.030 \times 10^{-6}$ \\
5 & $3.956 \times 10^{-10}$ & $5.367 \times 10^{-10}$ & $5.743 \times 10^{-9}$ & $7.054 \times 10^{-9}$ & $9.681 \times 10^{-9}$ \\
10 & $8.221 \times 10^{-13}$ & $1.098 \times 10^{-12}$ & $4.710 \times 10^{-11}$ & $5.738 \times 10^{-11}$ & $7.794 \times 10^{-11}$ \\
20 & $1.630 \times 10^{-15}$ & $2.172 \times 10^{-15}$ & $3.727 \times 10^{-13}$ & $4.531 \times 10^{-13}$ & $6.138 \times 10^{-13}$ \\
50 & $4.300 \times 10^{-19}$ & $5.715 \times 10^{-19}$ & $6.129 \times 10^{-16}$ & $7.445 \times 10^{-16}$ & $1.008 \times 10^{-15}$ \\
100 & $8.404 \times 10^{-22}$ & $1.117 \times 10^{-21}$ & $4.791 \times 10^{-18}$ & $5.819 \times 10^{-18}$ & $7.877 \times 10^{-18}$ \\
\hline
\end{tabular}

$$
W_{L 2}(x)=\sqrt{2 \pi x}\left(\frac{x}{e}\right)^{x}\left[x \sinh \left(\frac{1}{x}+\frac{1}{810 x^{7}}-\frac{67}{42,525 x^{9}}\right)\right]^{x / 2} .
$$

We have

$$
\begin{aligned}
& \lim _{x \rightarrow \infty} \frac{\ln \Gamma(x+1)-\ln W_{L 1}(x)}{x^{-7}}=-\frac{67}{85,050}, \\
& \lim _{x \rightarrow \infty} \frac{\ln \Gamma(x+1)-\ln W_{L 2}(x)}{x^{-9}}=\frac{19}{17,010} .
\end{aligned}
$$

These, in combination with Corollaries 2, 1 and 3, show that the approximation formula $W_{02}(x)$ given by (1.9) is the best among those listed above, which can be seen from comparison Table 1.

\section{Conclusion}

In this paper, we provide four Windschitl type approximation formulas for the gamma function, and prove that those functions, involving the gamma function and Windschitl type functions, have good properties, including monotonicity and convexity. From these facts we obtain some new sharp Windschitl type bounds for the gamma and factorial functions. These sharp inequalities, together with numerical comparisons, illustrate that $W_{02}(x)$ defined by (1.9) is the best approximation formula among those mentioned in Sect. 5 .

Moreover, we give a simple proof of Alzer's inequalities (1.4), and improve and strengthen Lu et al.'s inequalities (1.6).

It is worth mentioning that our proofs of Theorems $1-5$ are subtle and interesting, since the approximations deal with the gamma and hyperbolic sine functions, and it is difficult to establish their monotonicity and convexity by usual methods. Evidently, Lemmas 2 and 3 play important roles.

\section{Acknowledgements}

The authors would like to express their sincere thanks to the anonymous referees for their great efforts to improve this paper.

\section{Funding}

This work was supported by the Fundamental Research Funds for the Central Universities (No. 2015ZD29) and the Higher School Science Research Funds of Hebei Province of China (No. Z2015137).

\section{Competing interests}

The authors declare that they have no competing interests. 


\section{Author details}

'College of Science and Technology, North China Electric Power University, Baoding, P.R. China. ${ }^{2}$ Department of Science and Technology, State Grid Zhejiang Electric Power Company Research Institute, Hangzhou, P.R. China.

\section{Publisher's Note}

Springer Nature remains neutral with regard to jurisdictional claims in published maps and institutional affiliations.

Received: 3 March 2018 Accepted: 30 September 2018 Published online: 05 October 2018

\section{References}

1. Ramanujan, S.: The Lost Notebook and Other Unpublished Papers. Springer, Berlin (1988)

2. Burnside, W.: A rapidly convergent series for log N!. Messenger Math. 46, 157-159 (1917)

3. Gosper, R.W.: Decision procedure for indefinite hypergeometric summation. Proc. Natl. Acad. Sci. USA 75, 40-42 (1978). https://doi.org/10.1073/pnas.75.1.40

4. Alzer, H.: On some inequalities for the gamma and psi functions. Math. Comput. 66(217), 373-389 (1997). https://doi.org/10.1090/S0025-5718-97-00807-7

5. Shi, X., Liu, F., Hu, M.: A new asymptotic series for the Gamma function. J. Comput. Appl. Math. 195, 134-154 (2006) https://doi.org/10.1016/j.cam.2005.03.081

6. Batir, N.: Inequalities for the gamma function. Arch. Math. 91, 554-563 (2008). https://doi.org/10.1007/s00013-008-2856-9

7. Batir, N.: Bounds for the Gamma function. Results Math. 72, 865-874 (2017) https://doi.org/10.1007/s00025-017-0698-0

8. Mortici, C.: A new Stirling series as continued fraction. Numer. Algorithms 56(1), 17-26 (2011). https://doi.org/10.1007/s11075-010-9370-4

9. Mortici, C.: Improved asymptotic formulas for the gamma function. Comput. Math. Appl. 61, 3364-3369 (2011). https://doi.org/10.1016/j.camwa.2011.04.036

10. Mortici, C.: Further improvements of some double inequalities for bounding the gamma function. Math. Comput. Model. 57, 1360-1363 (2013). https://doi.org/10.1016/j.mcm.2012.11.020

11. Mortici, C.: A continued fraction approximation of the gamma function. J. Math. Anal. Appl. 402, 405-410 (2013). https://doi.org/10.1016/j.jmaa.2012.11.023

12. Mortici, C.: A new fast asymptotic series for the gamma function. Ramanujan J. 38, 549-559 (2015). https://doi.org/10.1007/s11139-014-9589-0

13. Nemes, G.: New asymptotic expansion for the Gamma function. Arch. Math. (Basel) 95, 161-169 (2010). https://doi.org/10.1007/s00013-010-0146-9

14. Nemes, G.: More accurate approximations for the gamma function. Thai J. Math. 9(1), 21-28 (2011)

15. Guo, B.N., Zhang, Y.J., Qi, F.: Refinements and sharpenings of some double inequalities for bounding the gamma function. J. Inequal. Pure Appl. Math. 9(1), Article ID 17 (2008)

16. Qi, F.: Integral representations and complete monotonicity related to the remainder of Burnside's formula for the gamma function. J. Comput. Appl. Math. 268, 155-167 (2014). https://doi.org/10.1016/j.cam.2014.03.004

17. Feng, L., Wang, W.: Two families of approximations for the gamma function. Numer. Algorithms 64, $403-416$ (2013). https://doi.org/10.1007/s11075-012-9671-x

18. Chen, C.-P.: Unified treatment of several asymptotic formulas for the gamma function. Numer. Algorithms 64 , 311-319 (2013). https://doi.org/10.1007/s11075-012-9667-6

19. Chen, C.-P.: Asymptotic expansions of the gamma function related to Windschitl's formula. Appl. Math. Comput. 245, 174-180 (2014). https://doi.org/10.1016/j.amc.2014.07.080

20. Chen, C.-P., Paris, R.B.: Inequalities, asymptotic expansions and completely monotonic functions related to the gamma function. Appl. Math. Comput. 250, 514-529 (2015). https://doi.org/10.1016/j.amc.2014.11.010

21. Chen, C.-P.: A more accurate approximation for the gamma function. J. Number Theory 164, 417-428 (2016). https://doi.org/10.1016/j.jnt.2015.11.007

22. Yang, Z.-H., Chu, Y.-M.: Asymptotic formulas for gamma function with applications. Appl. Math. Comput. 270 665-680 (2015). https://doi.org/10.1016/j.amc.2015.08.087

23. Yang, Z.-H.: Approximations for certain hyperbolic functions by partial sums of their Taylor series and completely monotonic functions related to gamma function. J. Math. Anal. Appl. 441, 549-564 (2016). https://doi.org/10.1016/j.jmaa.2016.04.029

24. Yang, Z.-H., Tian, J.-F.: Monotonicity and inequalities for the gamma function. J. Inequal. Appl. 2017, Article ID 317 (2017). https://doi.org/10.1186/s13660-017-1591-9

25. Yang, Z.-H., Tian, J.-F.: An accurate approximation formula for gamma function. J. Inequal. Appl. 2018, Article ID 56 (2018). https://doi.org/10.1186/s13660-018-1646-6

26. Lu, D.: A new sharp approximation for the Gamma function related to Burnside's formula. Ramanujan J. 35(1), 121-129 (2014). https://doi.org/10.1007/s11139-013-9534-7

27. Lu, D., Song, L., Ma, C.: A generated approximation of the gamma function related to Windschitl's formula. J. Number Theory 140, 215-225 (2014). https://doi.org/10.1016/j.jnt.2014.01.023

28. Lu, D., Song, L., Ma, C.: Some new asymptotic approximations of the gamma function based on Nemes' formula, Ramanujan's formula and Burnside's formula. Appl. Math. Comput. 253, 1-7 (2015). https://doi.org/10.1016/j.amc.2014.12.077

29. Xu, A., Hu, Y., Tang, P.: Asymptotic expansions for the gamma function. J. Number Theory 169, 134-143 (2016) https://doi.org/10.1016/j.jnt.2016.05.020

30. Qi, F., Niu, D.-W., Guo, B.-N.: Monotonic properties of differences for remainders of psi function. Int. J. Pure Appl. Math. Sci. 4(1), 59-66 (2007)

31. Guo, S., Qi, F.: A class of completely monotonic functions related to the remainder of Binet's formula with applications. Tamsui Oxf. J. Inf. Math. Sci. 25(1), 9-14 (2009) 
32. Shi, X.-Q., Liu, F.-S., Qu, H.-M.: The Burnside approximation of gamma function. Anal. Appl. 8(3), 315-322 (2010). https://doi.org/10.1142/S0219530510001643

33. Qi, F., Guo, B.-N.: Some properties of extended remainder of Binet's first formula for logarithm of gamma function. Math. Slovaca 60(4), 461-470 (2010). https://doi.org/10.2478/s12175-010-0025-7

34. Qi, F., Guo, B.-N.: Integral representations and complete monotonicity of remainders of the Binet and Stirling formulas for the gamma function. Rev. R. Acad. Cienc. Exactas Fís. Nat., Ser. A Mat. 111(2), 425-434 (2017). https://doi.org/10.1007/s13398-016-0302-6

35. Yang, Z.-H., Tian, J.-F.: Two asymptotic expansions for gamma function related to Windschitl's formula. Open Math. 16 1048-1060 (2018). https://doi.org/10.1515/math-2018-0088

36. Smith, W.D.: The gamma function revisited. http://schule.bayernport.com/gamma/gamma05.pdf (2006)

37. Alzer, H.: Sharp upper and lower bounds for the gamma function. Proc. R. Soc. Edinb. 139A, 709-718 (2009) https://doi.org/10.1017/S0308210508000644

38. Yang, Z.-H., Chu, Y.-M., Zhang, X.-H.: Sharp bounds for psi function. Appl. Math. Comput. 268, 1055-1063 (2015). https://doi.org/10.1016/j.amc.2015.07.012

39. Yang, Z.-H., Chu, Y.-M., Tao, X.-J.: A double inequality for the trigamma function and its applications. Abstr. Appl. Anal. 2014, Article ID 702718 (2014). https://doi.org/10.1155/2014/702718

40. Yang, Z.-H., Tian, J.: Monotonicity and sharp inequalities related to gamma function. J. Math. Inequal. 12(1), 1-22 (2018). https://doi.org/10.7153/jmi-2018-12-01

41. Yang, Z.-H., Tian, J.: Convexity and monotonicity for the elliptic integrals of the first kind and applications. arXiv:1705.05703 [math.CA]

42. Yang, Z.-H., Qian, W.-M., Chu, Y.-M., Zhang, W.: On approximating the arithmetic-geometric mean and complete elliptic integral of the first kind. J. Math. Anal. Appl. 462, 1714-1726 (2018). https://doi.org/10.1016/j.jmaa.2018.03.005

43. Tian, J., Wang, W., Cheung, W.-S.: Periodic boundary value problems for first-order impulsive difference equations with time delay. Adv. Differ. Equ. 2018, Article ID 79 (2018). https://doi.org/10.1186/s13662-018-1539-5

44. Tian, J.-F.: Triple Diamond-Alpha integral and Hölder-type inequalities. J. Inequal. Appl. 2018, Article ID 111 (2018). https://doi.org/10.1186/s13660-018-1704-0

45. Tian, J.-F., Ha, M.-H.: Extensions of Hölder's inequality via pseudo-integral. Math. Probl. Eng. 2018, Article ID 4080619 (2018). https://doi.org/10.1155/2018/4080619

46. Abramowttz, M., Stegun, I.A.: Handbook of Mathematical Functions with Formulas, Graphs and Mathematical Tables. Dover, New York (1972). https://doi.org/10.1063/1.3047921

\section{Submit your manuscript to a SpringerOpen ${ }^{\circ}$ journal and benefit from:}

- Convenient online submission

- Rigorous peer review

- Open access: articles freely available online

- High visibility within the field

- Retaining the copyright to your article

Submit your next manuscript at $\mathbf{s p r i n g e r o p e n . c o m ~}$ 